\title{
Mesoscale simulations of hydrodynamic squirmer interactions
}

\author{
Ingo O. Götze* and Gerhard Gompper ${ }^{\dagger}$ \\ Theoretical Soft Matter and Biophysics, Institut für Festkörperforschung and Institute for Advanced Simulation, \\ Forschungszentrum Jülich, 52425 Jülich, Germany
}

(Received 22 June 2010; revised manuscript received 30 September 2010; published 26 October 2010)

\begin{abstract}
The swimming behavior of self-propelled microorganisms is studied by particle-based mesoscale simulations. The simulation technique includes both hydrodynamics and thermal fluctuations that are both essential for the dynamics of microswimmers. The swimmers are modeled as squirmers, i.e., spherical objects with a prescribed tangential surface velocity, where the focus of thrust generation can be tuned from pushers to pullers. For passive squirmers (colloids), we show that the velocity autocorrelation function agrees quantitatively with the Boussinesq approximation. Single active squirmers show a persistent random-walk behavior, determined by forward motion, lateral diffusion, and orientational fluctuations, in agreement with theoretical predictions. For pairs of squirmers, which are initially swimming in parallel, we find an attraction for pushers and a repulsion for pullers, as expected. The hydrodynamic force between squirmer pairs is calculated as a function of the center-to-center distances $d_{c m}$ and is found to be consistent with a logarithmic distance dependence for $d_{c m}$ less than about two sphere diameters; here, the force is considerably stronger than expected from the far-field expansion. The dependence of the force strength on the asymmetry of the polar surface velocity is obtained. During the collision process, thermal fluctuations turn out to be very important and to strongly affect the postcollision velocity directions of both squirmers.
\end{abstract}

DOI: 10.1103/PhysRevE.82.041921

PACS number(s): 87.17.Jj, 47.63.Gd, 47.57.-s

\section{INTRODUCTION}

Self-propelled swimming enables microorganisms to explore their surroundings in order to find regions with higher nutrient concentration [1], to seek for or to avoid environmental conditions such as light [2], and to hunt or to escape from predators $[3,4]$. The evolutionary benefits of motility can thus outweigh the associated metabolic costs substantially [5-7]. Microswimmers are ubiquitous in aqueous environments - they have adapted to various habitats such as ponds and oceans and soil or the fluid interiors of animals (both hostile and symbiotic), and a variety of different propulsion mechanisms has evolved [8-10]. Because of their significance for many biological processes, a detailed understanding of their behavior is desirable. For example, swimming bacteria are involved in the spreading of infectional diseases, motile algae and protozoa play a vital role in the ecological system, and spermatozoa are indispensable for reproduction. Moreover, insights gained from swimming microorganisms may be useful for the development of nanorobots, e g., in medicine for targeted drug delivery or to remove clots in blood vessels.

Swimming at small length scales is characterized by low Reynolds numbers, where viscous forces dominate inertial forces, and propulsion mechanisms are required that are fundamentally different from those known from the macroscopic world [7]. One class of microswimmers are almost spherical organisms that are propelled by active hairlike organelles (cilia) covering the body. On a mesoscopic length scale, the synchronized beating (metachronal waves) of the cilia can be mapped onto a spherical envelope $[11,12]$, and

\footnotetext{
*i.goetze@fz-juelich.de

†g.gompper@fz-juelich.de
}

its time average corresponds to a sphere with a steady prescribed tangential surface velocity. Moreover, these objects — called "squirmers" - may also serve as a simple generic model for other types of microswimmers, for example, diffusiophoretic particles (such as polystyrene or silica beads with metallic caps $[13,14])$. To cope with the biodiversity of real microswimmers, the characteristics of the model can be varied from pullers to pushers, i.e., whether the thrust is generated mainly in the front or rear part of the body. This model was employed in Refs. $[15,16]$ to study monolayers of squirmers without thermal fluctuations or a single squirmer in the presence of thermal fluctuations, respectively.

While the understanding of the propulsion mechanisms of single microswimmers has considerably advanced $[1,7,8,17]$, much less is know about the hydrodynamic interactions that govern their collective behavior. As the hydrodynamic interactions are long ranged, the motion of microswimmers is strongly coupled in concentrated solutions, leading to dynamical phenomena such as bundling of spermatozoa $[18,19]$, bioconvection $[9,20]$, instabilities and pattern formation [21], or the graceful "dance" of Volvox algae [22]. Although other mechanisms such as chemotaxis can also be involved, hydrodynamic interactions play a crucial role for the collective behavior of swimming microorganisms $[23,24]$. The hydrodynamic interactions of microswimmers have been studied experimentally $[25,26]$ and theoretically $[10,15,18,25-29]$. However, most of these studies neglect the influence of thermal fluctuations and often rely on far-field approximations. In the present paper, we study the hydrodynamic interactions of pairs of squirmers that are small enough to be subject to thermal translational and rotational diffusion. We demonstrate that the thermal motion has a large effect on the effective interactions between two swimmers and their behavior after collisions.

Due to the inherent many-body character and the longranged nature of hydrodynamic interactions, mesoscale hy- 
drodynamic simulation techniques are particularly well suited to study the collective behavior of squirmer suspensions in the presence of thermal fluctuations. Here, we model the fluid by multiparticle collision dynamics (MPC) [30-32], which allows a quantitative description of the hydrodynamic behavior of self-propelled particles. Since MPC is an offlattice particle-based hydrodynamics simulation technique, the incorporation of moving boundaries is straightforward and thermal fluctuations are naturally included [31,32]. Moreover, a combination with a molecular-dynamics algorithm for the squirmer motion is easily possible. Here, we consider the interaction between pairs of squirmers; however, due to the high computational efficiency of MPC, the model is also well suited for the investigation of larger systems involving many squirmers.

\section{MODEL}

\section{A. Squirmer mode}

The squirmer is modeled as a hard sphere of radius $R$ (and diameter $\sigma$ ) with a prescribed tangential surface velocity $\mathbf{v}_{\mathrm{sq}}$, causing a propulsion in the direction of squirmer's instantaneous orientation $\hat{\mathbf{e}}$. The relative velocity at a surface point $\mathbf{r}_{\mathrm{s}}$ (with respect to squirmer's center) is given by [12]

$$
\mathbf{v}_{\mathrm{sq}}\left(\mathbf{r}_{\mathrm{s}}, \hat{\mathbf{e}}\right)=\sum_{n=1}^{2} B_{n} \frac{2}{n(n+1)}\left(\frac{\hat{\mathbf{e}} \cdot \mathbf{r}_{\mathrm{s}}}{R} \frac{\mathbf{r}_{\mathrm{s}}}{R}-\hat{\mathbf{e}}\right) P_{n}^{\prime}\left(\frac{\hat{\mathbf{e}} \cdot \mathbf{r}_{\mathrm{s}}}{R}\right),
$$

where $P_{n}^{\prime}(\cos \theta)$ is the derivative of the $n$th Legendre polynomial and $B_{n}$ is the amplitude of the $n$th mode of the surface velocity. The absolute local surface velocity of the squirmer is given by

$$
\mathbf{v}_{\mathrm{s}}\left(\mathbf{r}-\mathbf{r}_{\mathrm{c}}, \hat{\mathbf{e}}\right)=\mathbf{v}_{\mathrm{c}}+\mathbf{v}_{\mathrm{sq}}\left(\mathbf{r}-\mathbf{r}_{\mathrm{c}}, \hat{\mathbf{e}}\right)+\boldsymbol{\Omega} \times\left(\mathbf{r}-\mathbf{r}_{\mathrm{c}}\right),
$$

where $\mathbf{r}_{\mathrm{c}}, \mathbf{v}_{\mathrm{c}}$, and $\boldsymbol{\Omega}$ are the sphere position, velocity, and angular velocity, respectively. The constant $B_{1}$ sets the average velocity of the squirmer, $v=\left\langle\hat{\mathbf{e}} \cdot \mathbf{v}_{\mathrm{c}}\right\rangle=\frac{2}{3} B_{1}[10]$. Changing the ratio of the two squirming modes, $\beta=B_{2} / B_{1}$, allows for adjusting the characteristics of the model from pullers $(\beta$ $>0)$ to pushers $(\beta<0)$.

\section{B. Fluid model}

The fluid is modeled by MPC, a particle-based mesoscale hydrodynamics simulation technique [30-32]. Here, the fluid is represented by $N$ point particles of mass $m$ that undergo alternating streaming and collision steps. In the streaming step, the particles do not interact with each other and propagate according to

$$
\mathbf{r}_{\mathrm{i}}(t+\Delta t)=\mathbf{r}_{\mathrm{i}}(t)+\mathbf{v}_{\mathrm{i}}(t) \Delta t,
$$

where $\Delta t$ is the time interval between collisions. Subsequently, the particles are sorted into the cells of a simple cubic lattice with lattice constant $a$; the origin of this lattice is randomly shifted before each collision step to ensure Galilean invariance [33]. The collision step then mimics the simultaneous interaction of all particles within each cell. There are several versions of the collision algorithm, which all have in common that the linear momentum is conserved in each collision cell. Here, we employ a variant that also conserves angular momentum locally, denoted as $\mathrm{MPC}-\mathrm{AT}+a$ [34]. The new velocities are then given by

$$
\begin{aligned}
\mathbf{v}_{i}^{\prime}= & \mathbf{v}_{\mathrm{c}}^{\mathrm{G}}+\mathbf{v}_{i}^{\mathrm{ran}}-\sum_{j \in \text { cell }} \mathbf{v}_{j}^{\mathrm{ran}} / N_{\mathrm{c}}+m \boldsymbol{\Pi}^{-1} \sum_{j \in \text { cell }}\left\{\mathbf{r}_{j, \mathrm{c}} \times\left(\mathbf{v}_{j}-\mathbf{v}_{j}^{\mathrm{ran}}\right)\right\} \\
& \times \mathbf{r}_{i, \mathrm{c}},
\end{aligned}
$$

where $\boldsymbol{\Pi}$ is the moment-of-inertia tensor of the particles in the cell and $N_{\mathrm{c}}$ is the number of particles in a cell. The relative position is $\mathbf{r}_{i, \mathrm{c}}=\mathbf{r}_{i}-\mathbf{r}_{\mathrm{c}}^{\mathrm{G}}$, where $\mathbf{r}_{\mathrm{c}}^{\mathrm{G}}$ is the center of mass of the particles in the cell and $\mathbf{v}_{\text {ran }}$ are random velocities chosen from a Maxwell-Boltzmann distribution at temperature $T$. This collision method serves at the same time as a thermostat. It has been shown that it is essential to guarantee local angular momentum conservation within the fluid in studies of rotating colloids [35] and of coexisting fluids with different viscosities [35,36].

\section{Boundary conditions}

The implementation of no-slip boundary conditions in the streaming step can be achieved by the bounce-back rule [37]. In contrast to fixed walls considered in Ref. [37], the boundary conditions on the surface of a moving sphere require a small modification. The new velocities of fluid particles that hit the sphere during the streaming are then given by

$$
\mathbf{v}_{\mathrm{i}}^{\prime}=-\mathbf{v}_{\mathrm{i}}+2 \mathbf{v}_{\mathrm{s}},
$$

where $\mathbf{v}_{S}$ is the surface velocity at the point of impact according to Eq. (2). The change in the linear and angular momenta is transferred to the squirmer.

The implementation of the coupling of the fluid to the squirmer in the collision step needs more sophistication. To minimize slip at surfaces of objects, virtual particles are required in cells that overlap with boundaries [37], which are of particular importance for small time steps $\Delta t$ [38], where the collisional contribution to the viscosity dominates. Due to the occurrence of the inertia tensor in the collision algorithm MPC-AT $+a$, the use of explicit virtual particles is necessary here, as described in Ref. [35]. To this end, a layer of thickness $\sqrt{3}$ underneath the squirmer surface is filled randomly with virtual particles before each collision step, where the total number of virtual particles is chosen such that their average density in that layer equals the density of the fluid. In order to preset the fluid velocity at the surface according to Eq. (2), we assign velocities $\mathbf{v}_{\text {ran }}+\mathbf{v}_{\mathrm{s}}$ to the virtual particles before the collision step, where $\mathbf{v}_{\text {ran }}$ are random velocities selected from a Maxwell-Boltzmann distribution at the same temperature $T$ as the fluid's and $\mathbf{v}_{\mathrm{s}}$ is the surface velocity of the squirmer at the same azimuthal and polar angles. These virtual particles then participate in the collision step. This method has been shown to yield very good agreement with the theoretical velocity field in Ref. [16].

The change in linear momentum experienced by the virtual particles is transferred to the squirmer. In addition, a torque originating from the coupling of the fluid to the squirmer via the virtual particles is exerted on the squirmer during the collision step. Therefore, the change in the angu- 
lar momentum of the virtual particles (with respect to the squirmer's center) has to be transferred to the squirmer, too (regardless of whether a locally angular-momentumconserving version of MPC is used or not). Thus, in the collision step, the rotational velocity of the colloid is updated according to

$$
\mathbf{\Omega}^{\prime}=\boldsymbol{\Omega}+I^{-1} \sum_{i=1}^{N_{\text {virt }}}\left(\mathbf{r}_{i}-\mathbf{r}_{\mathrm{c}}\right) \times\left(\mathbf{v}_{\mathrm{i}}^{\prime}-\mathbf{v}_{\mathrm{i}}\right),
$$

where $N_{\text {virt }}$ is the number of virtual particles and $I$ is the moment of inertia of the squirmer. We would like to emphasize the importance of the torque on the squirmer arising from angular momentum conservation on the length scale of the squirmer size during the collision step due to the coupling via the virtual particles, since its negligence leads to serious errors. We will return to this point in Sec. III B below.

\section{Parameters}

We choose different sets of parameters for the simulations of single-squirmer systems and of squirmer pairs. The squirmer mass is chosen such that its mass density equals that of the fluid.

For the systems with two squirmers, we choose a fluid density $\rho=\left\langle N_{\mathrm{c}}\right\rangle a^{-3}=10$ and a time step $\delta t=0.01 t_{0}$, where $t_{0}$ $=a \sqrt{m / k_{B} T}$. For our choice of parameters, the kinematic viscosity of the fluid is $\nu=3.589 a \sqrt{k_{B} T / m}$. The squirmer has a radius $R=4 a$.

In order to obtain realistic hydrodynamic interactions between squirmers, their velocities must be much smaller than the velocity of propagation of the hydrodynamic interactions. The time it takes the momentum to diffuse a characteristic distance $d$, e.g., the colloid separation, is the kinematic time $t_{\text {kin }}=d^{2} / \nu[39,40]$. For the squirmer pairs, we use an initial surface-to-surface separation $d_{s}=8 a$. This sets limitations for the squirmer velocity $v \ll \nu / d_{s}=0.45 a / t_{0}$. However, if $v$ is too small, thermal fluctuations prevail over the hydrodynamic interactions we are interested in. Here, we mainly study squirmers with $B_{1}=0.08 a / t_{0}$, which implies $v=2 / 3 B_{1}$ $=0.053 a / t_{0}$, corresponding to a Reynolds number $\mathrm{Re}$ $=v \sigma / \nu=0.12$ and a Péclet number $\mathrm{Pe}=v \sigma / D=1155$, where $\sigma$ is the sphere diameter and $D$ is its translational diffusion constant. In order to investigate the effect of thermal fluctuations, we also investigate the interactions of slower squirmers with $B_{1}=0.06 a / t_{0}, B_{1}=0.04 a / t_{0}$, and $B_{1}=0.02 a / t_{0}$, which correspond to Péclet numbers $\mathrm{Pe}=866, \mathrm{Pe}=577$, and $\mathrm{Pe}$ $=289$, respectively. The typical values of the Péclet number for biological and synthetic squirmers can range from $\mathcal{O}(10)$ for diffusiophoretic particles [13] to $\mathcal{O}\left(10^{2}\right)$ for bacillus subtilis, a flagellated rod-shaped bacterium (with a length of $4 \mu \mathrm{m}$, a swimming velocity of $v \simeq 10 \mu \mathrm{m} / \mathrm{s}$ [23], and a diffusion constant estimated from Stokes friction of $D$ $\simeq 0.1 \mu \mathrm{m}^{2} / \mathrm{s}$ [41]), as well as ciliates or Volvox algae [42]. As we will see later, the rotational diffusion plays a crucial role for the behavior of the squirmers. Therefore, we define also a rotational Péclet number $\mathrm{Pe}_{\mathrm{r}}=v /\left(\sigma D_{r}\right)=385$, where $D_{r}=k_{B} T /\left(\pi \eta \sigma^{3}\right)$ is the rotational diffusion constant. In real systems, the magnitude of the rotational Péclet number is mainly determined by the sphere diameter $\sigma$.

For the single-passive-sphere and single-squirmer systems, the fluid density is $\rho=5$ and the time step is $\delta t$ $=0.05 t_{0}$. This choice of parameters corresponds to a kinematic viscosity $\nu=0.6375 a \sqrt{k_{B} T / m}$. We use a smaller sphere radius $R=3 a$ and a lower viscosity here (compared to the two-squirmer systems) to obtain a high rotational diffusion in order to facilitate the measurement of the orientation autocorrelation functions. Here, we set $B_{1}=0.04 a / t_{0}$; this corresponds to a Reynolds number $\operatorname{Re}=0.5$ and a rotational Péclet number $\mathrm{Pe}_{\mathrm{r}}=19$.

In the following, lengths are usually measured in units of the squirmer diameter $\sigma$ and times are in units of $\sigma / v$, i.e., the time needed for a squirmer to travel its own diameter. For passive colloids, time is measured in units of the rotational diffusion constant.

\section{RESULTS}

\section{A. Single passive sphere}

Since the squirmer model is based on spherical objects impenetrable for fluid particles, we first investigate the dynamics of a single (passive) colloidal particle immersed in an MPC fluid. This corresponds to the special case $B_{n}=0$ for all $n$. We measure the velocity autocorrelation function (VACF),

$$
C_{V}(t)=\langle\mathbf{V}(t) \cdot \mathbf{V}(0)\rangle
$$

and the angular velocity autocorrelation function (AVACF),

$$
C_{\Omega}(t)=\langle\boldsymbol{\Omega}(t) \cdot \boldsymbol{\Omega}(0)\rangle .
$$

The values of the autocorrelation function for $t=0$ follow directly from the equipartition theorem of equilibrium statistical mechanics,

$$
C_{V}(0)=3 k_{B} T / M, \quad C_{\Omega}(0)=3 k_{B} T / I,
$$

where $M$ is the mass and $I$ is the moment of inertia of the colloid. In the long-time limit, mode coupling theory predicts an algebraic decay $[43,44]$,

$$
\begin{gathered}
\lim _{t \rightarrow \infty} C_{V}(t)=\frac{k_{B} T}{4 \pi \rho}[\pi(\nu+D) t]^{-3 / 2}, \\
\lim _{t \rightarrow \infty} C_{\Omega}(t)=\frac{3 \pi k_{B} T}{\rho}[4 \pi(\nu+D) t]^{-5 / 2} .
\end{gathered}
$$

At smaller time scales, the VACF is expected to follow the Boussinesq approximation [45] (based on the analysis of the incompressible Navier-Stokes equation)

$$
\begin{aligned}
C_{V}(t)= & \frac{3 k_{B} T}{2 \pi R^{3} \rho\left(5-8 \rho^{\prime} / \rho\right)}\left(\frac{R^{2}}{\nu}\right)^{1 / 2}\left[a_{+} \exp \left(a_{+}^{2} t\right) \operatorname{erfc}\left(a_{+} \sqrt{t}\right)\right. \\
& \left.-a_{-} \exp \left(a_{-}^{2} t\right) \operatorname{erfc}\left(a_{-} \sqrt{t}\right)\right]
\end{aligned}
$$




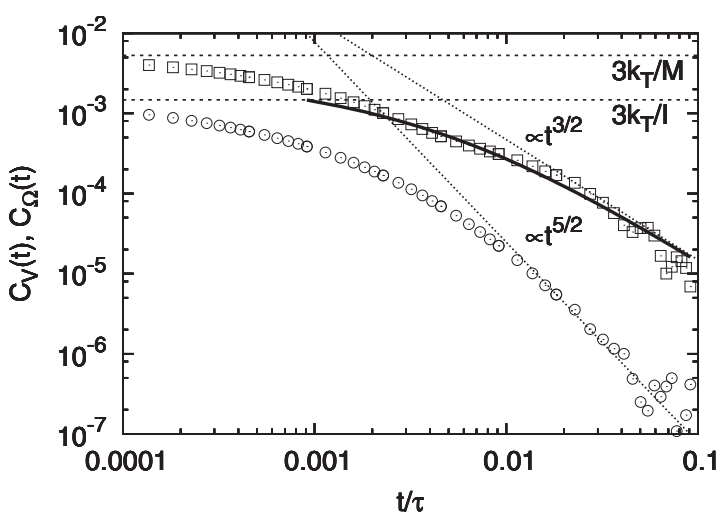

FIG. 1. Velocity autocorrelation function ( $\square$ ) and angular velocity autocorrelation function $(\bigcirc)$ of a single passive colloid from simulation, compared with the theoretical expectation at $t=0$ from equipartition theorem (dashed lines) and the respective algebraic long-time decays (dotted lines) as well as the Boussinesq approximation (full line).

where

$$
a_{ \pm}=\frac{3}{2}\left(\frac{\nu}{R^{2}}\right)^{1 / 2}\left[3 \pm\left(5-8 \frac{\rho^{\prime}}{\rho}\right)^{1 / 2}\right] /\left(1+2 \frac{\rho^{\prime}}{\rho}\right) \text {, }
$$

and $\rho^{\prime}$ is the mass density of the sphere (in our simulations, $\left.\rho^{\prime}=\rho\right)$.

In Fig. 1, the simulation results for $C_{V}(t)$ and $C_{\Omega}(t)$ are shown together with the theoretical predictions of Eqs. (10)-(12). At $t=0$, the simulation data coincide with the equipartition theorem (with only about $1.5 \%$ and $0.25 \%$ deviations, respectively). There is excellent agreement of the AVACF with the asymptotic power-law prediction for times $t / \tau>0.02$, with the characteristic time scale $\tau=1 /\left(2 D_{r}\right)$ of rotational diffusion. Furthermore, the VACF is well described by the Boussinesq approximation for times $t / \tau$ $>0.002$. In both cases, there are no adjustable parameters.

We want to briefly comment on the differences of our approach with previous studies of rotational diffusion of passive colloids with no-slip boundary conditions [40,46-48]. In most of these studies, thermal or bounce-back boundary conditions are employed without virtual wall particles. It was pointed out in Ref. [48] that thermal boundary conditions generate considerable wall slip. Bounce-back boundary conditions are suitable to generate a good approximation to noslip surfaces without virtual particles, but only for relatively large time steps $h \geq 0.1[37,48]$. This comes at the cost of a non-negligible Enskog contribution to the diffusion coefficient $[46,47]$, so that diffusion is not dominated by hydrodynamics. Furthermore, when large forces are acting on a colloidal particle, a time step $h \geq 0.1$ is often not sufficient to produce a high enough viscosity and to guarantee low Reynolds numbers. All these complications can be avoided by the coupling of the fluid to the colloid via virtual particles, as shown above. No noticeable Enskog contribution appears because of the small time step and the collisions with the virtual particles.

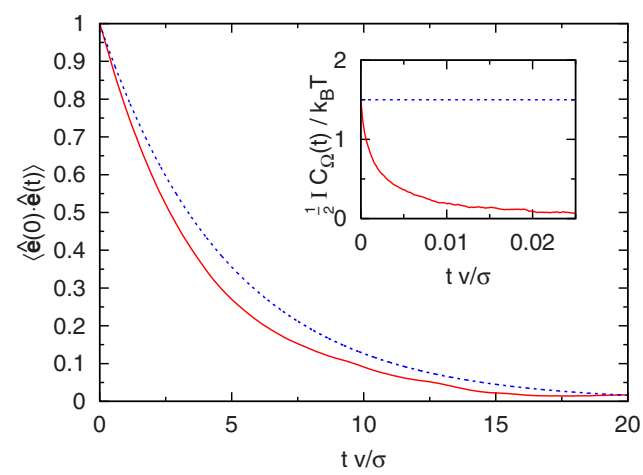

FIG. 2. (Color online) Orientation autocorrelation function $\langle\hat{\mathbf{e}}(0) \cdot \hat{\mathbf{e}}(t)\rangle$ for a single squirmer, with Péclet number $\mathrm{Pe}=1155$. The full line shows the simulation data, while the dashed line corresponds to the theoretical result $\exp \left(-2 D_{r} t\right)$. The inset shows the angular velocity autocorrelation function $C_{\Omega}(t)=\langle\boldsymbol{\Omega}(0) \cdot \boldsymbol{\Omega}(t)\rangle$. The dashed line represents the theoretical value at $t=0$.

\section{B. Single squirmers}

Next, we test the coupling to the fluid for a single active squirmer. It has already been shown in Ref. [16] with the stochastic-rotation dynamics version of MPC that the theoretically calculated fluid velocity field around the squirmer [12] is reproduced quite well in MPC simulations, when virtual particles that are used are given the velocity of the squirmer surface at the same polar angle. This is in agreement with our observations employing MPC-AT $+a$.

However, the rotational energy $\frac{1}{2} I\langle\boldsymbol{\Omega} \cdot \boldsymbol{\Omega}\rangle$ of a single squirmer was found in Ref. [16] to exceed the theoretical value $\frac{3}{2} k_{B} T$ by about a factor of 6 when virtual particles are used. Moreover, in Ref. [16] the orientation autocorrelation function $\langle\hat{\mathbf{e}}(0) \cdot \hat{\mathbf{e}}(t)\rangle$ displays an exponential decay with a characteristic time scale $\tau$. This time scale is predicted theoretically to be $\tau=1 /\left(2 D_{r}\right)$, where $D_{r}$ is the rotational diffusion constant, the same as for a passive colloid [39]. However, the value of $\tau$ that can be read off from Fig. 5(b) of Ref. [16], where $\langle\hat{\mathbf{e}}(0) \cdot \hat{\mathbf{e}}(t)\rangle$ has decayed to $1 / e$, is by orders of magnitude smaller than its theoretical value $\tau=1 /\left(2 D_{r}\right)$ $=4 \pi \eta R^{3} / k_{B} T$ computed for the parameters given in Ref. [16].

We are only able to reproduce these drastic discrepancies if we inadmissibly neglect the torque on the squirmer that arises in the collision step. During the collision step, the fluid and the rotating colloid also exchange angular momentum via the coupling with the virtual particles. Thus, the change in the linear and angular momenta of the virtual particles has to be transferred to the colloid, regardless of whether a locally angular-momentum-conserving version of MPC (which concerns angular momentum conservation in the fluid on the scale of the collision-box size) is employed or not. In order to obtain quantitatively correct results, however, a version with angular momentum conservation is required.

The orientational autocorrelation function measured for a single squirmer in a box of size $5 \sigma \times 5 \sigma \times 5 \sigma$ with periodic boundary conditions is plotted in Fig. 2 together with the theoretical result $\langle\hat{\mathbf{e}}(0) \cdot \hat{\mathbf{e}}(t)\rangle=\exp \left(-2 D_{r} t\right)$. Here, we observe a deviation in $\tau$ of about $20 \%$ from the theoretical value $\tau$ 


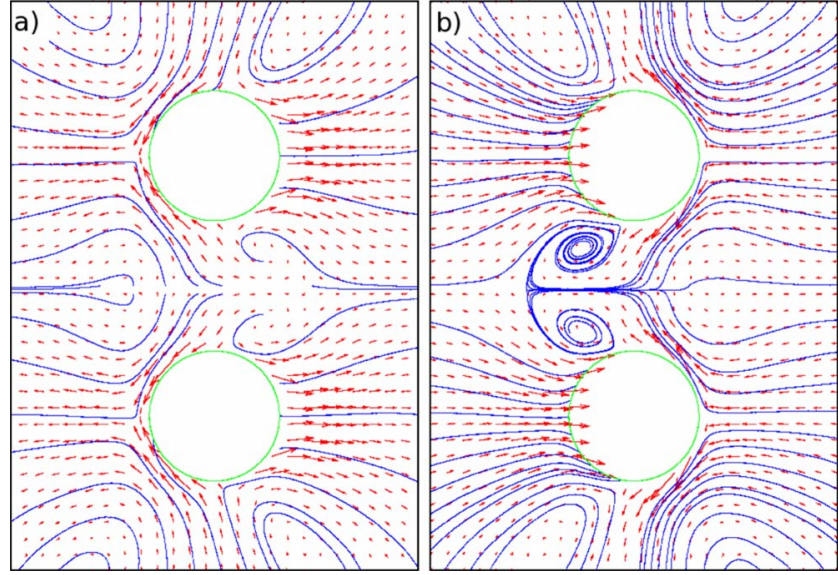

FIG. 3. (Color online) Velocity fields for fixed parallel pairs of squirmers, for (a) pusher (with $\beta=-3$ ) and (b) puller (with $\beta=+3$ ), with Péclet number $\mathrm{Pe}=1155$. Swimmers move to the right. Streamlines serve as a guide to the eye. Only a fraction of the simulation box is shown. (Due to the finite resolution of the measures velocity field, some streamlines end on the squirmers' surfaces.)

$=4 \pi \eta R^{3} / k_{B} T$ for a radius $R=3 a$. We attribute this small deviation to discretization effects due to size of the collision boxes, as the agreement improves with increasing squirmer radius and thus decreasing $a / R$. With a correct implementation of angular momentum conservation, as described in Sec. II $\mathrm{C}$ above, the rotational energy is in perfect agreement with the expected value (see the inset of Fig. 2).

\section{Pairs of squirmers}

First, we study the flow field around a pair of squirmers at fixed positions with a surface-to-surface distance $d_{s}=\sigma$ and a fixed parallel orientation. The size of the simulation box is $10 \sigma$ in the direction of the connection of the squirmer centers and $6.25 \sigma$ in both perpendicular directions. Periodic boundary conditions are employed in all three dimensions. As MPC naturally includes thermal fluctuations, the time average over many simulation steps has to be taken in order to obtain the average flow field with good accuracy. Subsequently, the total average fluid velocity is subtracted to obtain the flow field in the laboratory frame. The resulting velocity fields in the plane that contains the squirmers' centers are shown in Fig. 3 for pusher and puller. In the region between the two squirmers, we observe a convergent flow for the pullers $(\beta=+3)$; thus, fluid is pressed into the gap, increasing the pressure there and leading to repulsion. On the other hand, we find a divergent flow for the pushers $(\beta$ $=-3$ ), i.e., a mutual suction of the squirmers toward each other. To make this clearer, we also show some streamlines. For the pullers, we observe convergence of the streamlines in the region between the squirmers, some of them ending in vortices. Since the flow field is three dimensional, fluid can leave the plane under consideration in the perpendicular direction.

Moreover, we measure the interaction force of fixed parallel oriented squirmers for various center-to-center distances $d_{c m}$ (see Fig. 4). The forces are normalized with the Stokes

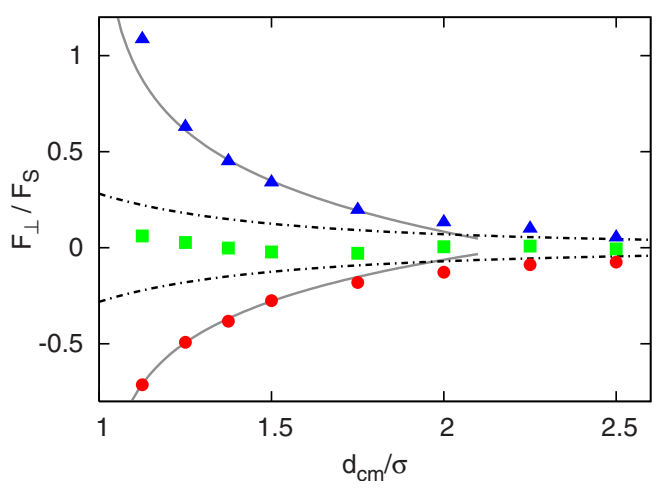

FIG. 4. (Color online) Interaction force of two parallel squirmers (in units of the corresponding Stokes force at the same swimming velocity) as a function of the center-to-center distance $d_{c m}$ for $\beta=-3$ (red circles), $\beta=0$ (green squares), and $\beta=+3$ (blue triangles), with Péclet number $\mathrm{Pe}=1155$. Dashed-dotted lines indicate the expected asymptotic $\left(\sigma / d_{c m}\right)^{2}$ law for large distances, while full gray lines indicate the logarithmic decay of Eq. (14) at distances $d_{c m} / \sigma-1 \ll 1$ with $C=0.2$.

force acting on a sphere moving with the same velocity $v$, i.e., $F_{S}=6 \pi \eta R v$. In agreement with the above considerations, we find repulsive forces $F_{\perp}$ for puller and an attraction for pusher. In both cases, the force for $d_{c m} / \sigma \lesssim 1.7$ is well described by a logarithmic dependence separation $d_{c m}$,

$$
F_{\perp, \text { near }}=A_{0}(\beta) F_{S}\left[\ln \left(\frac{d_{c m}}{\sigma}-1\right)+C\right] .
$$

This functional dependence has been derived in Ref. [10] in a near-field expansion. In the opposite limit of large distances, a far-field approximation predicts a power-law decay of the fluid velocity $[10,12]$,

$$
u_{z, \mathrm{far}}=\frac{1}{16} \beta B_{1}\left(\frac{\sigma}{d_{c m}}\right)^{2},
$$

generated by one swimmer (moving in the $x$ direction) at the position of the other swimmer (displaced by $d_{c m}$ in the $z$ direction). This implies a force

$$
F_{\perp, \mathrm{far}}=3 \pi \sigma u_{z, \mathrm{far}} .
$$

The simulation results presented in Fig. 4 show that the data approach the asymptotic behavior (16) for $d_{c m}>2 \sigma$. It is important to note that the hydrodynamic interactions on distances $d_{c m} \lesssim 2 \sigma$ are considerably stronger than predicted by the far-field result. The neutral $(\beta=0)$ squirmers, on the other hand, show only a very weak interaction with alternating sign as a function of $d_{c m}$.

The dependence of the interaction force on $\beta$ for a fixed separation $d_{c m}=1.5 \sigma$ is plotted in Fig. 5. We find a simple linear dependence. This implies that in Eq. (14), the amplitude of the force is given by

$$
A_{0}(\beta)=0.10 \beta+0.02 \text {. }
$$

It is interesting to note that the magnitude of the hydrodynamic interaction at distance $d_{c m} / \sigma=1.5$ and $\beta=5$ has about the same magnitude as the Stokes drag force $F_{S}$. 


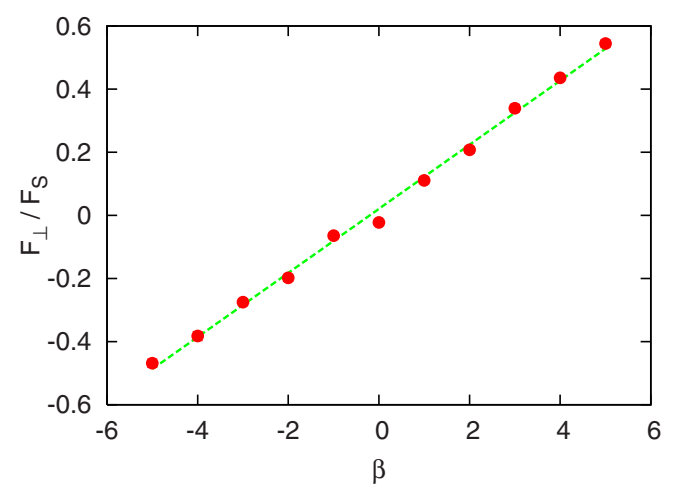

FIG. 5. (Color online) Interaction force of two parallel squirmers (in units of the corresponding Stokes force at the same swimming velocity) as a function of ratio $\beta$ of surface velocity modes, for center-to-center distance $d_{c m}=1.5 \sigma$ and Péclet number $\mathrm{Pe}$ $=1155$. The dashed green line is a guide to the eye.

Next, we allow the squirmers to swim freely, starting with two squirmers at center-to-center distance $d_{c m}=2 \sigma$ and parallel initial orientation. For $\beta=-3,0,3$, we use 200 independent runs each to determine the time evolution of the distance distribution shown in Fig. 6. The distribution is characterized by a peak, which drifts and broadens due to the translational diffusion, as well as the rotational diffusion and thus reorientation of the swimming direction. Figure 6 demonstrates again that pushers, pullers, and neutral swimmers show a qualitatively different behavior. The peak shifts to larger distances for pullers $(\beta=+3)$, i.e., they repel each other, while the peak shifts to smaller distances for pushers $(\beta=-3)$, i.e., they attract each other. For $\beta=0$ the position of the peak does not change.

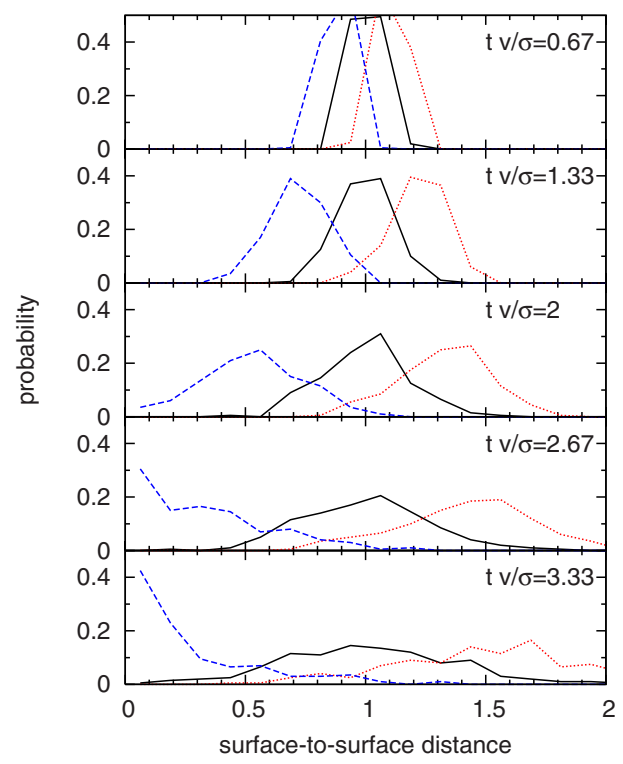

FIG. 6. (Color online) Normalized histograms of surface-tosurface distances $d_{s} / \sigma$ of squirmer pairs at various times, determined from many independent runs. The initial configurations at $t$ $=0$ are identical to those in Fig. 3. Blue dashed lines represent the simulation results for $\beta=-3$ (pusher), black full lines for $\beta=0$ (neutral squirmer), and red dotted lines for $\beta=+3$ (puller). In all cases, the Péclet number is $\mathrm{Pe}=1155$.

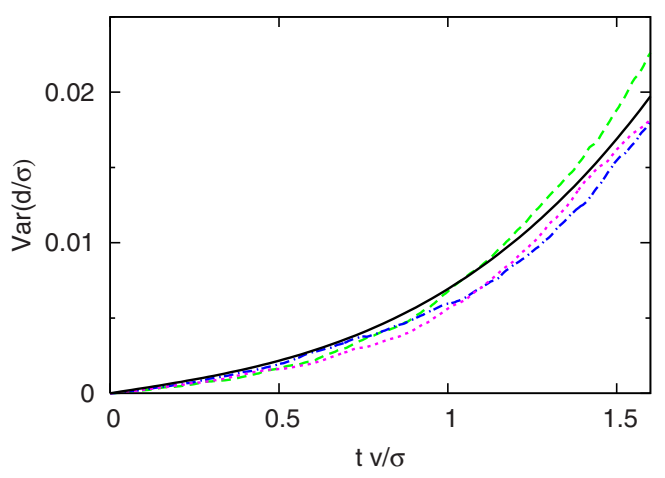

FIG. 7. (Color online) Variance of the center-to-center distances, $\operatorname{Var}(d / \sigma)=\sigma^{-2}\left\langle\left[d_{c m}-\bar{d}_{c m}(t)\right]^{2}\right\rangle$, as a function of time for $\beta=-3$ (dashed green line), $\beta=0$ (dashed-dotted blue line), and $\beta=+3$ (dotted red line). In all cases, the Péclet number is $\mathrm{Pe}=1155$. The full black line shows the theoretical prediction (18).

From the distributions, we calculate the variance of the center-to-center distances $d_{c m}=d_{s}+\sigma$ as a function of time (see Fig. 7). The contribution of the translational diffusion of two squirmers is $\left\langle\left[d_{c m}-\bar{d}_{c m}(t)\right]^{2}\right\rangle=4 D t$, where $\bar{d}_{c m}(t)$ is the average distance at time $t$. The contribution due to the rotational diffusion of the two squirmer can be calculated straightforwardly from the orientational correlation function $\langle\hat{\mathbf{e}}(0) \cdot \hat{\mathbf{e}}(t)\rangle=\exp \left(-2 D_{r} t\right)$, as follows directly from the theory of semiflexible polymers $[13,50,51]$ [the trajectory of a squirmer with only rotational diffusion is equivalent to the conformation of a semiflexible polymer with "persistence length" $\left.\tau=1 /\left(2 D_{r}\right)\right]$. In the limit $D_{r} t \ll 1$, the leading contri-

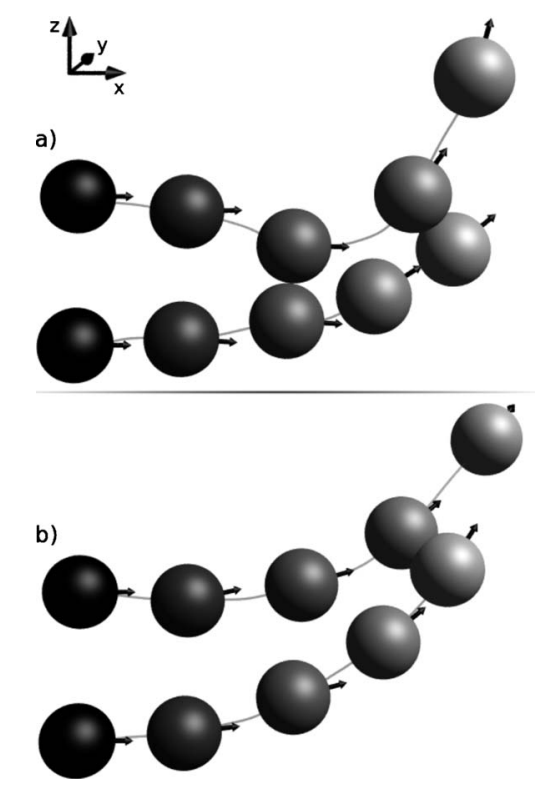

FIG. 8. Sequences of snapshots for pairs of initially parallel pushers at fixed time intervals $\Delta t v / \sigma=1.67$ for two different realizations of the thermal noise, with Péclet number $\mathrm{Pe}=1155$. The initial parallel offset is $\Delta x=0$. The instantaneous squirmer orientations are indicated by the arrows. (a) Squirmers touch at time $t v / \sigma \simeq 3.33$, but later depart again. (b) Squirmers do not touch. Movies of the motion of interacting squirmer pairs are provided in Ref. [49]. 

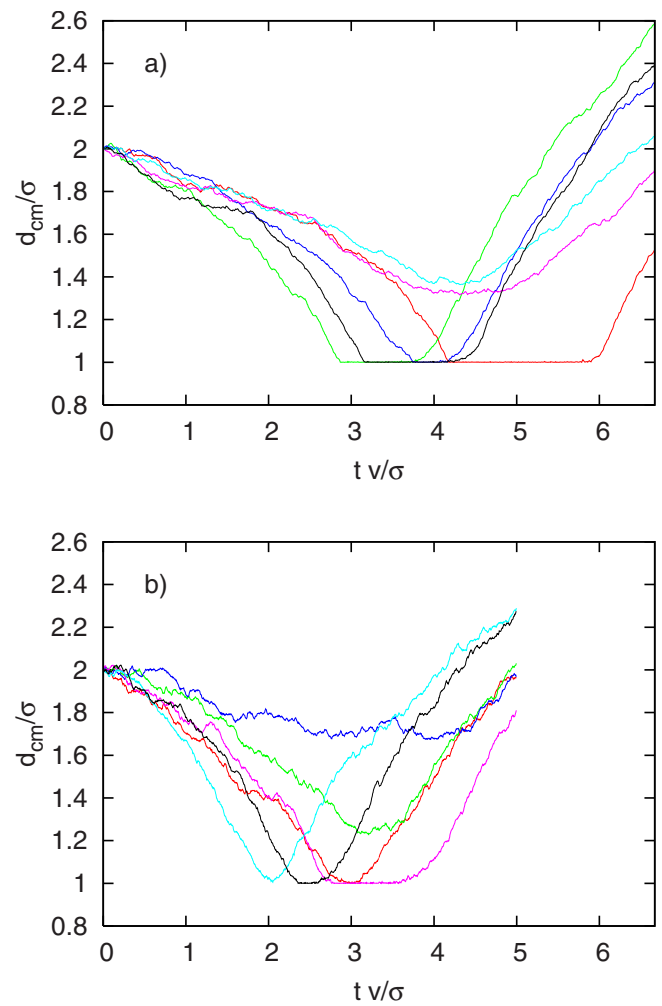

FIG. 9. (Color online) Time-dependent distance $d_{c m}$ of pairs of initially parallel pushers $(\beta=-3)$ with initial parallel offset $\Delta x=0$, for (a) $\mathrm{Pe}=1155$ with swimming velocity $v=0.053 a / t_{0}$ and (b) $\mathrm{Pe}$ $=577$ with swimming velocity $v=0.027 a / t_{0}$.

bution to the variance is here $\frac{2}{3} v^{2} D_{r} t^{3}$. Thus, the time evolution of the variance is expected to be

$$
\left\langle\left[d_{c m}-\bar{d}_{c m}(t)\right]^{2}\right\rangle=4 D t+v^{2} \frac{4}{3} D_{r} t^{3}+\mathcal{O}\left(\left(D_{r} t\right)^{4}\right)
$$

This theoretical prediction is in excellent agreement with our simulation results, as shown in Fig. 7-again without any adjustable parameters. By equating the first and second terms in Eq. (18), it is easy to see that translational diffusion dominates for $t v / \sigma<1$, while orientational diffusion is more important for $t v / \sigma>1$, i.e., after the squirmer has traveled a distance of its own diameter.

Two typical trajectories of pushers which are initially oriented in parallel, overlaid with stroboscopic snapshots, are shown in Fig. 8. The time-dependent distances of several representative trajectories are presented in Fig. 9(a). First, pushers move toward each other. After a time $t v / \sigma \simeq 3.33$, it is very likely to find a pair of pushers at contact [see Fig. 8(a)]; however, although there is an attractive force between parallel pushers, due to orientational fluctuations, their collision is not inevitable [see Fig. 8(b)]. As a consequence of thermal fluctuations, one squirmer lags behind the other in this case, which weakens the attraction or even turns into a repulsion. We observe that in most of the 200 independent runs, the pairs of pushers stick together for a while after contact. However, at later times, the squirmers reorient themselves and the trajectories subsequently diverge-despite the attractive interaction between pushers swimming in parallel.

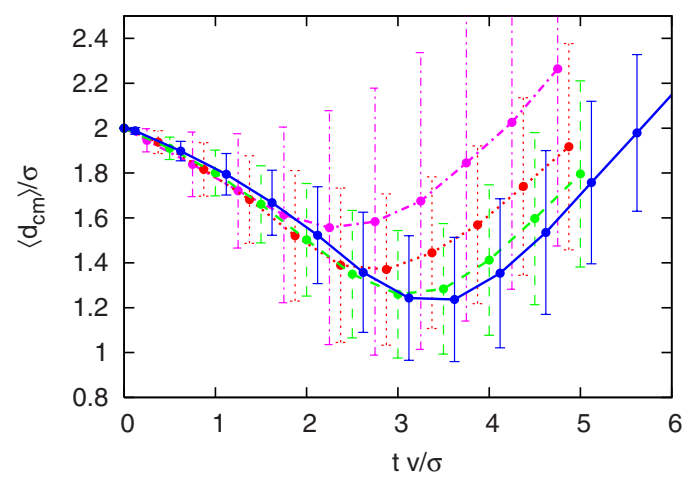

FIG. 10. (Color online) Average distance of squirmer pairs (with $\beta=-3$ ) as a function of scaled time for different swimming velocities. The corresponding Péclet numbers are $\mathrm{Pe}=289$ (magenta dashed-dotted line), 577 (red dotted line), 866 (green dashed line), and 1155 (blue full line). The error bars represent the standard deviation.

In order to study the effect of thermal fluctuations, we vary the swimming velocity and thus the Péclet number. In Fig. 9(b), the time-dependent distance of squirmer pairs is shown for six independent runs, where the swimming velocity has been reduced by a factor of 2 compared to Fig. 9(a). As expected, an increased spreading of the trajectories for independent runs can be seen. Moreover, we observe shorter contact times since the increased fluctuations facilitate the reorientation of the squirmers.

In Fig. 10, the time evolution of the average interpair distance is shown for four different swimming velocities, obtained from 64 independent runs each. Without thermal fluctuations, all pairs would have contact at the same time at a minimum distance of $d_{\min }=\sigma$. It can be extrapolated from Fig. 10 that the first-contact time in the absence of fluctuations occurs approximately at $t v / \sigma=4$. With thermal fluctuations, independent trajectories show an increasing spreading with decreasing Péclet numbers, which is reflected in an increasing standard deviation of the distance distribution. At short times $(t v / \sigma \lesssim 2)$ the average distance is dominated by the mutual hydrodynamic interaction and depends only very weakly on Pe (for the Péclet numbers Pe $\gtrsim 290$ studied here). It should be noticed that at constant $\beta$, the perpendicular force $F_{\perp}$ increases linearly with the swimming velocity [see Eqs. (14)-(16)]; on the other hand, the time needed to traverse a given distance is proportional to $1 / v$. Therefore, the pair separation as a function of the rescaled time should

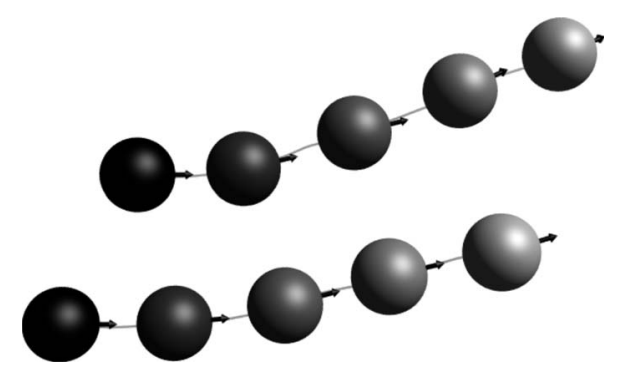

FIG. 11. Same as Fig. 8, but with an initial offset $\Delta x=\sigma, \Delta z$ $=2 \sigma$. The time interval is $\Delta t v / \sigma=1.67$ Movies of the motion of interacting squirmer pairs are provided in Ref. [49]. 


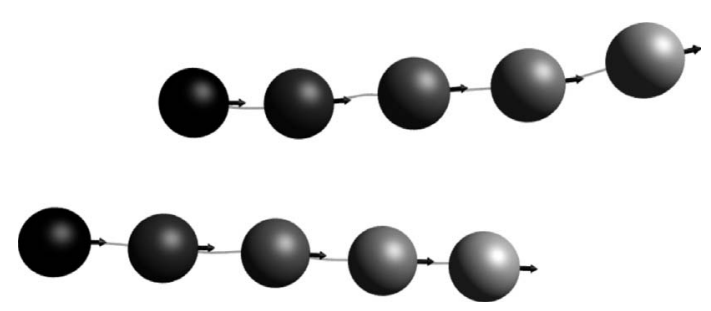

FIG. 12. Same as Fig. 8, but with an initial offset $\Delta x=2 \sigma, \Delta z$ $=2 \sigma$. The time interval is $\Delta t v / \sigma=1.67$.

be independent of $v$-in the absence of fluctuations. At larger times $(t v / \sigma \geq 2)$, fluctuations become relevant. As the trajectories tend to fan out more for smaller Pe, the probability of squirmer pairs to touch decreases. The minimal distance increases and is shifted to shorter times with decreasing Péclet number, because first contact can occur earlier, contact times are shorter, and contact probability decreases, so that more trajectories diverge already at shorter times.

We would like to point out that if there is less than one collision cell of fluid between two surfaces, hydrodynamic interactions are inaccurately represented by MPC. However, since in the case of touching spheres this condition is only violated very locally close to the point of contact, we expect the qualitative behavior to be predicted correctly in our simulations.

To corroborate the observation that the squirmer orientation after collisions (or near collisions) for initial parallel offset $\Delta x=0$ is very sensitive to thermal fluctuations, we modify the initial configuration such that the two parallel oriented pushers start with an offset $\Delta x \neq 0, \Delta z=2 \sigma$ (see Fig. 11 for $\Delta x=\sigma$ and Fig. 12 for $\Delta x=2 \sigma$ ). For an initial configuration with parallel offset $\Delta x=\sigma$, we observe a deflection of both pushers in the positive $z$ direction. For $\Delta x=2 \sigma$, instead of an attraction, a weak repulsion is observed.

To study this behavior more quantitatively, the average angle $\alpha$ of the squirmers orientation with the $x$ axis after a time $t v / \sigma=6.67$ is plotted in Fig. 13 as a function of the initial parallel offset $\Delta x$. For symmetry reasons, the average angle for $\Delta x=0$ must vanish. On the other hand, for very large $\Delta x$, the interaction vanishes, which again implies a zero average angle. We observe a maximum of the deflection angle at a small offset in the range $0<\Delta x<0.5 \sigma$. In Ref. [10], a similar problem has been studied (but without thermal fluctuations) for pullers, which are deflected in the opposite sense, i.e., in the direction of the squirmer that lags behind.

The variance of the scattering angle $\alpha$ is not due to an insufficient number of independent runs, but reflects the width of the distribution of scattering angles. Figure $13 \mathrm{dem}-$ onstrates that the variance becomes very large for $|\Delta x| \rightarrow 0$, because when the two squirmers collide, small changes in the relative orientation and position have a drastic effect on the postcollisional angles. For example, in the case without parallel offset, a squirmer pair may collide or not, as we have seen in Fig. 9; in fact, for $\Delta x=0$ the distribution of scattering angles is bimodal, with peaks at $\alpha \simeq \pm 45^{\circ}$. Also for a finite parallel offset, the importance of thermal fluctuations is reflected in the large variance in Fig. 13.

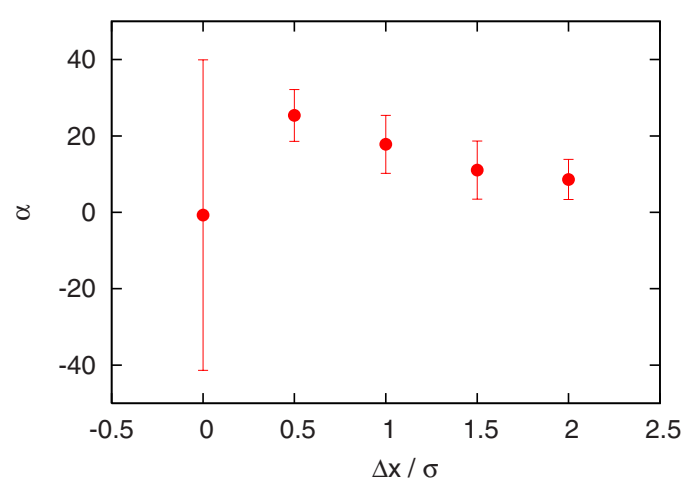

FIG. 13. (Color online) Average deflection angle $\alpha$ after a time $t v / \sigma=6.67$ for pairs of pushers $(\beta=-3)$, with Péclet number Pe $=1155$ and initial vertical offset $\Delta z=2 \sigma$, as a function of the parallel offset $\Delta x$. Error bars represent the standard deviations.

\section{CONCLUSIONS}

We have investigated the swimming behavior of single squirmers and squirmer pairs by mesoscale hydrodynamics simulations. The results show that multiparticle collision dynamics (MPC) is able to describe squirmer systems quantitatively when the correct boundary conditions are used and when angular momentum conservation is fully implemented. The advantage of the MPC method is that it includes both hydrodynamic interactions and thermal fluctuations consistently and is computationally very efficient.

The motion of a single squirmer with thermal fluctuations is determined by forward motion and translational diffusion and by orientational fluctuations of the swimming direction [13]. We find that on times scales $t<\sigma / v$, when the squirmer has traveled less than its diameter, forward motion with translational diffusion dominates, while at later times, orientational fluctuations lead to a persistent random-walk behavior with persistence length $\xi_{p}=v /\left(2 D_{r}\right)$.

For two squirmers with initially parallel orientation, we find that the interaction depends on the mode ratio $\beta$ of surface velocities, with a repulsion for pullers and an attraction for pushers, in agreement with theoretical expectations. The simulation results for the hydrodynamic force between two squirmers with distances $d_{c m} / \sigma<2$ follow a logarithmic distance dependence, as predicted theoretically [10]. The force amplitude is obtained to be a linear function of the mode ratio $\beta$.

As initially parallel pushers approach each other due to hydrodynamic attractions, they can eventually collide. This process strongly depends on thermal fluctuations. In same cases, collisions may be completely avoided. In most other cases, the exit angles after the collision show a wide distribution. Furthermore, postinteraction angles are found to strongly depend on the initial lateral offset.

We conclude that the interactions of squirmers are mainly determined by the short-range hydrodynamic interactions. For larger distances, hydrodynamic forces are weak and thermal fluctuations of the swimming directions dominate. The importance of thermal fluctuations of course increases with decreasing Péclet number. Our results show that the MPC approach is well suited for quantitative simulation studies of multisquirmer systems. 


\section{ACKNOWLEDGMENTS}

Stimulating discussions with Jens Elgeti, Gerhard Nägele,
Roland G. Winkler, and Yingzi Yang are gratefully acknowledged.
[1] H. C. Berg, E. Coli in Motion (Springer, New York, 2004).

[2] W. Nultsch and D.-P. Häder, Photochem. Photobiol. 47, 837 (1988).

[3] K. Jürgens and C. Matz, Antonie van Leeuwenhoek 81, 413 (2002).

[4] R. Guerrero, C. Pedrós-Alió, I. Esteve, J. Mas, D. Chase, and L. Margulis, Proc. Natl. Acad. Sci. U.S.A. 83, 2138 (1986).

[5] J. A. Raven and K. Richardson, New Phytol. 98, 259 (1984).

[6] D. W. Crawford, Microb. Ecol. 24, 1 (1992).

[7] E. M. Purcell, Am. J. Phys. 45, 3 (1977).

[8] E. Lauga and T. R. Powers, Rep. Prog. Phys. 72, 096601 (2009).

[9] T. J. Pedley and J. O. Kessler, Annu. Rev. Fluid Mech. 24, 313 (1992).

[10] T. Ishikawa, M. P. Simmonds, and T. J. Pedley, J. Fluid Mech. 568, 119 (2006).

[11] M. J. Lighthill, Commun. Pure Appl. Math. 5, 109 (1952).

[12] J. R. Blake, J. Fluid Mech. 46, 199 (1971).

[13] J. R. Howse, R. A. L. Jones, A. J. Ryan, T. Gough, R. Vafabakhsh, and R. Golestanian, Phys. Rev. Lett. 99, 048102 (2007).

[14] A. Erbe, M. Zientara, L. Baraban, C. Kreidler, and P. Leiderer, J. Phys.: Condens. Matter 20, 404215 (2008).

[15] T. Ishikawa and T. J. Pedley, Phys. Rev. Lett. 100, 088103 (2008).

[16] M. T. Downton and H. Stark, J. Phys.: Condens. Matter 21, 204101 (2009).

[17] M. J. Lighthill, Mathematical Biofluiddynamics (SIAM, Philadelphia, 1975).

[18] Y. Yang, J. Elgeti, and G. Gompper, Phys. Rev. E 78, 061903 (2008).

[19] F. Hayashi, J. Insect Physiol. 42, 859 (1996).

[20] J. R. Platt, Science 133, 1766 (1961).

[21] D. Saintillan and M. J. Shelley, Phys. Rev. Lett. 100, 178103 (2008).

[22] K. Drescher, K. C. Leptos, I. Tuval, T. Ishikawa, T. J. Pedley, and R. E. Goldstein, Phys. Rev. Lett. 102, 168101 (2009).

[23] C. Dombrowski, L. Cisneros, S. Chatkaew, R. E. Goldstein, and J. O. Kessler, Phys. Rev. Lett. 93, 098103 (2004).

[24] A. Sokolov, I. S. Aranson, J. O. Kessler, and R. E. Goldstein, Phys. Rev. Lett. 98, 158102 (2007).

[25] T. Ishikawa and M. Hota, J. Exp. Biol. 209, 4452 (2006).

[26] L. H. Cisneros, R. Cortez, C. Dombrowski, R. E. Goldstein, and J. O. Kessler, Exp. Fluids 43, 737 (2007).

[27] T. Ishikawa, G. Sekiya, Y. Imai, and T. Yamaguchi, Biophys. J. 93, 2217 (2007).

[28] G. P. Alexander, C. M. Pooley, and J. M. Yeomans, J. Phys.:
Condens. Matter 21, 204108 (2009).

[29] M. Ramia, D. L. Tullock, and N. Phan-Thien, Biophys. J. 65, 755 (1993).

[30] A. Malevanets and R. Kapral, J. Chem. Phys. 110, 8605 (1999).

[31] R. Kapral, Adv. Chem. Phys. 140, 89 (2008).

[32] G. Gompper, T. Ihle, D. M. Kroll, and R. G. Winkler, Adv. Polym. Sci. 221, 1 (2009).

[33] T. Ihle and D. M. Kroll, Phys. Rev. E 63, 020201(R) (2001).

[34] H. Noguchi, N. Kikuchi, and G. Gompper, EPL 78, 10005 (2007).

[35] I. O. Götze, H. Noguchi, and G. Gompper, Phys. Rev. E 76, 046705 (2007).

[36] S. Meßlinger, B. Schmidt, H. Noguchi, and G. Gompper, Phys. Rev. E 80, 011901 (2009).

[37] A. Lamura, G. Gompper, T. Ihle, and D. M. Kroll, EPL 56, 319 (2001).

[38] Y.-G. Tao, I. O. Götze, and G. Gompper, J. Chem. Phys. 128, 144902 (2008).

[39] J. K. G. Dhont, An Introduction to Dynamics of Colloids (Elsevier, Amsterdam, 1996).

[40] J. T. Padding and A. A. Louis, Phys. Rev. E 74, 031402 (2006).

[41] Our estimate of the diffusion constant of $B$. subtilis differs from the value $D=10^{3} \mu \mathrm{m}^{2} / \mathrm{s}$ given in Ref. [23], because we do not consider the active tumbling motion of these bacteria.

[42] M. B. Short, C. A. Solari, S. Ganguly, T. R. Powers, J. O. Kessler, and R. E. Goldstein, Proc. Natl. Acad. Sci. U.S.A. 103, 8315 (2006).

[43] M. H. Ernst, E. H. Hauge, and J. M. J. van Leeuwen, Phys. Rev. Lett. 25, 1254 (1970).

[44] E. H. Hauge and A. Martin-Löf, J. Stat. Phys. 7, 259 (1973).

[45] E. J. Hinch, J. Fluid Mech. 72, 499 (1975).

[46] J. T. Padding, A. Wysocki, H. Löwen, and A. A. Louis, J. Phys.: Condens. Matter 17, S3393 (2005).

[47] J. T. Padding and W. J. Briels, J. Chem. Phys. 132, 054511 (2010).

[48] J. K. Whitmer and E. Luijten, J. Phys.: Condens. Matter 22, 104106 (2010).

[49] See supplementary material at http://link.aps.org/supplemental/ 10.1103/PhysRevE.82.041921 for movies of the motion of pairs of squirmers, which are started with parallel orientation and lateral offsets $\Delta x=0$ and $\Delta x=\sigma$.

[50] J. Elgeti and G. Gompper, EPL 85, 38002 (2009).

[51] M. Doi and S. F. Edwards, The Theory of Polymer Dynamics (Clarendon Press, Oxford, 1986). 